Cahiers de philosophie de l'université de

\title{
De plus hautes diversités : la misanthropie et la futurologie de Gabriel Tarde
}

\section{David Toews}

Traducteur : Anne Devarieux et Thibaut Von Lennep

\section{OpenEdition Journals}

Édition électronique

URL : https://journals.openedition.org/cpuc/319

DOI : $10.4000 /$ cpuc.319

ISSN : 2677-6529

Éditeur

Presses universitaires de Caen

\section{Édition imprimée}

Date de publication : 31 décembre 2017

Pagination : 103-122

ISBN : 978-2-84133-859-7

ISSN : $1282-6545$

\section{Référence électronique}

David Toews, "De plus hautes diversités : la misanthropie et la futurologie de Gabriel Tarde », Cahiers de philosophie de l'université de Caen [En ligne], 54 | 2017, mis en ligne le 01 février 2019, consulté le 05 février 2023. URL : http://journals.openedition.org/cpuc/319 ; DOI : https://doi.org/10.4000/cpuc.319

\section{(@) $(1) \Theta$}

Creative Commons - Attribution - Pas d'Utilisation Commerciale 4.0 International - CC BY-NC 4.0 https://creativecommons.org/licenses/by-nc/4.0/ 


\section{De plus hautes diversités: la misanthropie et la futurologie de Gabriel Tarde}

En même temps que Français ou Anglais, nous sommes mammifères, et à ce titre nous roulons dans notre sang non seulement des germes d'instincts sociaux qui nous prédisposent à imiter nos semblables, à croire ce qu'ils croient, à vouloir ce qu'ils veulent, mais encore des ferments d'instincts non sociaux, parmi lesquels il s'en trouve d'antisociaux. Certes, si la société nous avait entièrement faits, elle ne nous aurait faits que sociables ${ }^{1}$.

Mais il se peut parfaitement que la civilisation s'arrête un jour pour se recueillir et enfanter, que le flux de l'imitation ait ses rivages, et que, par l'effet même de son déploiement excessif, le besoin de sociabilité diminue, ou plutôt s'altère et se transforme en une sorte de misanthropie générale, très compatible d'ailleurs avec une circulation commerciale modérée et une certaine activité d'échanges industriels réduits au strict nécessaire, mais surtout très propre à renforcer en chacun de nous les traits distinctifs de notre individualité intérieure. Alors éclora la plus haute fleur de la vie sociale, la vie esthétique, qui, exception si rare encore et si incomplète parmi nous, se généralisera en se consommant ${ }^{2}$.

$L^{\prime}$ A QUESTION DE LA CONSTITUTION D'Un SUJET COLLECTIF est le point focal de la pensée de Gabriel Tarde ${ }^{3}$. On le voit le plus clairement dans Monadologie et sociologie ${ }^{4}$, Les lois de l'imitation 5 mais aussi dans

1. G. Tarde, Monadology and Sociology, T. Lorenc (éd. et trad.), Melbourne, Re.Press, 2012, p. 47, en ligne à l'adresse suivante: http://re-press.org/book-files/9780980819724Monadology_and_Sociology.pdf.

2. G. Tarde, The Laws of Imitation, New York, H. Holt, 1903, p. 392.

3. É. Alliez, «Présentation: Tarde et le problème de la constitution», in G. Tarde, Monadologie et sociologie, Paris, Institut d'édition Sanofi-Synthélabo (Les empêcheurs de penser en rond - CEuvres de Gabriel Tarde, 1), 1999, p. 10-32.

4. G. Tarde, Monadology and Sociology.

5. G. Tarde, The Laws of Imitation. 
ses essais ( La croyance et le désir ${ }^{6}{ }^{\text {ou }}$ "L'opinion et la foule $»^{7}$ ), et enfin dans Les lois sociales ${ }^{8}$, ouvrage crucial dans lequel Tarde essaie de donner un cadre général à sa pensée. La constitution du collectif est le thème clé de ses investigations philosophiques s'agissant de Leibniz ${ }^{9}$ et de Maine de Biran ${ }^{10}$. La transition du rationalisme philosophique à l'école sociologique post-rationaliste de Tarde et Durkheim s'opère en excluant la notion de substance incapable d'être une solution pertinente au problème moderne des relations sociales ${ }^{11}$. Pourtant, si elles demeurent inadaptées, et incapables de nous convaincre avec l'idée de la conciliation des éléments de l'univers, indices d'expériences harmonieuses, les doctrines de la substance du début de la période moderne fournissent des indications sur le rôle crucial des résistances, des difficultés, et de la manière dont les autres «s'imposent» dans notre expérience ${ }^{12}$. On se souvient de la description faite par Leibniz de ces métaphysiciens amateurs qui dans le passé avaient l'habitude de présumer ce qui doit être expliqué ${ }^{13}$. Leibniz leur avait fait rapporter leurs marchandises à un maître horloger. Un tel expert aurait la compétence technique requise pour s'occuper d'elles. Et pourtant on ne peut pas s'empêcher d'imaginer Leibniz saisi d'ambivalence, quand on se représente le lieu de travail de l'artisan faiblement éclairé, modeste et confiné, en contraste avec l'activité des érudits scolastiques qui sans doute représentaient à cette époque le comble de la mobilité, de l'ouverture et de la flexibilité intellectuelles.

La vocation sociologique post-rationaliste de ces philosophes que sont Tarde et Durkheim, dont les débats ${ }^{14}$ forment le creuset d'une nouvelle forme de théorie sociale, tourne autour d'une question que la fin

6. G. Tarde, «Belief and Desire», in Gabriel Tarde on Communication and Social Influence, T. N. Clark (éd.), Chicago, University of Chicago Press, 2011, p. 195-208.

7. G. Tarde, "The Public and the Crowd», in Gabriel Tarde on Communication..., p. 277-296.

8. G. Tarde, Social Laws: An Outline of Sociology, Kitchener, Batoche Books, 20oo, en ligne à l'adresse suivante: $\mathrm{http}: / /$ socserv.mcmaster.ca/econ/ugcm/3ll $/$ /tarde/laws.pdf.

9. G. Tarde, Monadology and Sociology.

10. G. Tarde, Maine de Biran et l'évolutionnisme en psychologie, É. Alliez (éd.), A. Devarieux (préface), Paris, Institut d'édition Sanofi-Synthélabo (Les empêcheurs de penser en rond), 2000 .

11. D. Toews, "Tarde and Durkheim and the Non-Sociological Ground of Sociology ", in The Social after Gabriel Tarde: Debates and Assessments, M. Candea (dir.), Londres - New York, Routledge, 2010, p. 80-93.

12. Sur le thème de l'expérience comme «imposition", voir D. Toews, «The Impositions of Experience», in Rethinking the Human Sciences [conférence], University of Windsor, 2010, article accessible en ligne à l'adresse suivante: https://davidtoews.org/2017/11/23/ the-impositions-of-experience/.

13. G. W. Leibniz, Discourse on Metaphysics and Other Essays, D. Gerber, R. Ariew (éd.), Indianapolis, Hackett Publishing Company, 1991.

14. Pour la reconstruction du débat, voir G. Tarde, É. Durkheim, «The Debate», in The Social after Gabriel Tarde..., p. 85-98. 
du XIX ${ }^{e}$ siècle avait fini par mettre devant les yeux de chacun: doit-on stoïquement accepter dans toute son étendue la nouvelle réalité moderne des «fonctions assujettissantes et des redites monotones ${ }^{15}$ ? Sans l'harmonie préétablie, la diversité multi-englobante, parfaite et refermée sur elle-même, laisse la place à la scène de la division du travail bien moins illuminée et éclairée, et bien moins magistralement coordonnée. Au lieu d'une forme finale de diversité façonnée par Dieu, il y a désormais des diversités locales variées qui doivent être formées et négociées par les acteurs. Ou bien est-ce le confinement de l'homme moderne dans les cellules de ses propres occupations qui paraît plutôt ouvrir de larges espaces personnels à la créativité, comme dans l'image tardienne d'un système refuge de grottes magnifiques ${ }^{16}$ ? Tandis qu'il semble que Durkheim ne voie pas d'objection à maintenir la fonction sociale des illusions religieuses, la vision tardienne du futur paraît plutôt obéir à la déduction suivante: si la constitution du sujet collectif n'est plus nécessairement structurée comme un projet divin, la question devient alors autant celle du renversement des constitutions sociales que celle, liminaire, de la constitution même du sujet collectif. Dans le futur, semble-t-il, la fuite et l'insociabilité devront en quelque façon devenir des principes positifs et communément acceptés et non plus des pratiques furtives déviantes. De cette façon chacun adoptera les nouvelles «grottes» de l'ère moderne.

Cela - et la proximité de Tarde avec ce qui a été nommé le spiritualisme de Maine de Biran, le maître philosophique de Tarde, ou de Bergson, son «disciple» philosophique - soulève la question des choix de Tarde. Quel chemin essaie-t-il d'emprunter entre le réalisme social moderne d'un côté et la futurologie utopiste de l'autre? La diversité moderne est-elle une plus haute diversité, comme Tarde semble l'affirmer à plusieurs endroits de son œuvre ${ }^{17}$ ? Et quel est le rôle dévolu à l'ingéniosité humaine scientifique et technologique dans ce processus moderne de déconstitution et de reconstitution du collectif?

Ces questions peuvent, dans une certaine mesure, être simplifiées si on les rassemble sous la question fondamentale du comment. C'est une chose de parler de la constitution de la collectivité, c'en est une autre de comprendre comment une telle constitution est réellement dans les faits capable de fonctionner ou non. Et à la façon de Leibniz nous enjoignant d'éviter les

15. G. Tarde, The Laws of Imitation, p. 393.

16. Tel qu'il est décrit dans l'œuvre de fiction Fragment d'histoire future: Underground Man, Nabu Press, 2010. Une catastrophe solaire conduit les êtres humains à migrer vers des grottes souterraines et inaugure une nouvelle ère de l'autosuffisance et de l'innovation humaines.

17. G. Tarde, The Laws of Imitation, p. 393; G. Tarde, Monadology and Sociology, p. 39-47. 
erreurs des érudits médiévaux ${ }^{18}$ (auxquels Leibniz ajoute les physiciens, qui semblent aussi négliger la question du comment), comment tous ces corps, êtres humains, animaux et choses, en s'associant, vont-ils s'organiser? Comment interagiront-ils? Les questions nouvelles doivent aller au-delà du pur sociologisme, de l'affirmation du principe du collectif comme de la réaffirmation platonicienne de l'idéal de justice, et descendre jusqu'aux faits désordonnés de tous les détails sociologiques ou non - des sociabilités et insociabilités gênantes et incommodes, comme celles que Socrate avait dévoilées pour les tourner à ses propres fins dans ses "dialogues». Ces sociabilités et insociabilités intrusives doivent être désormais négociées et comprises comme plus fondamentales que le présupposé axial de Durkheim selon lequel il suffit de vivre comme si nous étions obligés de nous associer avec les autres ${ }^{19}$, ce qui, du point de vue de Tarde, revient purement et simplement au désir d'oublier la difficulté du comment.

La question du comment semble discutable si elle est traitée au niveau d'une métaphysique sociale post-rationaliste qui convertit les substances en forces actives, i. e. si elle est interprétée strictement comme ce qui doit être fait. Mais elle se révèle subitement comme l'essence de la question à partir du moment où l'on réalise que la socialité, quelque active, combattante et auto-déterminante qu'elle soit, n'est jamais automatiquement équivalente à la sociabilité. C'est ici que la plupart des observateurs font le saut dans le politique, en donnant au politique - ce qui doit être fait - l'air d'une science complémentaire de la métaphysique sociale. Mais Tarde - et c'est un point d'accord avec Durkheim - rechigne à faire ce pas.

Ainsi, observe Tarde, «malgré le morcellement politique [de la féodalité] qui, quoique bien amoindri, a subsisté, le nivellement social s'est refait» à l'échelle mondiale ${ }^{20}$. Tarde et Durkheim insistent sur le besoin moderne d'une pratique de la sociologie intermédiaire entre la collectivité et le régime politique. C'est en effet l'ouverture à la science et aux institutions de la modernité, que les deux penseurs ont vue, bien que de manière différente.

Un des messages clés de Monadologie et sociologie est que les pratiques de la sociologie et de la science convergent, théorie qui, si elle est correcte, entrerait en contradiction avec le désir de Durkheim que la sociologie soit purement et simplement une branche de la science. Quoi qu'il en soit, suivre le cheminement du raisonnement de Tarde à propos de la question de la constitution et déconstitution du sujet collectif, ce n'est pas simplement

18. Voir G. W. Leibniz, Discourse on Metaphysics...

19. É. Durkheim, The Rules of Sociological Method, S. Lukes (éd.), W. D. Halls (trad.), New York, The Free Press, 1982, p. 130.

20. G. Tarde, The Laws of Imitation, p. 391. 
comprendre l'imbrication de la science et de la société, comme si la seule description des assemblages élémentaires des deux, déplaçant ce qui est devenu la théorie durkheimienne de l'obligation sociale, pouvait épuiser le problème. On doit tenir compte du rôle central du sujet et de la manière dont il en est venu à participer positivement à la fois à la sociabilité et à l'insociabilité ${ }^{21}$. La figure de la fuite illustre ce rôle nouveau. Qui n'admettra pas qu'aujourd'hui le fait d'échapper à autrui soit aussi fondamental dans nos relations, que la confiance partagée dans les technologies et institutions communes? Une chose aussi simple que la conversation - Blanchot l'a fait valoir - requiert sur le plan éthique que l'on s'isole de l'autre, ce que les sociologues américains nomment la cérémonie de l'espace personnel ${ }^{22}$. Dans le monde contemporain, transformé en une vaste panoplie de médias de toutes formes, et façonné précisément par ce que Tarde a nommé les relations commerciales et les échanges économiques, il est particulièrement nécessaire, afin de comprendre la manière de réduire - et les possibilités de défier - cette forme du capitalisme digital, d'enquêter sur la nature changeante de l'insociabilité parallèlement à la sociabilité. Dans Les lois sociales, la théorie générale tardienne de la science implique la progression des diversités d'un plateau bas à un plateau plus haut, et contient la clé de compréhension - c'est ce que je soutiens - d'une nouvelle relation entre sociabilité et insociabilité, que nous commençons seulement à concevoir en sociologie. J'espère montrer qu'on peut la comprendre comme une forme de futurologie. Par "futurologie», j'entends une théorisation consciente et raisonnée spéculativement du futur. La futurologie de Tarde, pourtant, n'est ni utopique ni dystopique, et ce point - telle est ma thèse - est étroitement lié à sa théorie du développement de la science.

\section{Le concept tardien de "diversités plus hautes"}

Le XIX ${ }^{\mathrm{e}}$ siècle, et sa célébration de la modernité, passe pour la première période dans laquelle s'est imposée au grand nombre l'idée qu'un vaste changement dans la vision humaine du monde était devenu nécessaire et irréversible. Ce qui semblait garantir cette nécessité était l’avancement de la science. On voit apparaître dans cette période la notion de vision du

21. D. Toews, «Tarde and Durkheim and the Non-Sociological Ground...»; D. Toews, «Tarde's Sociology of Difference: Its Classical Roots and Contemporary Meanings », Journal of Classical Sociology, vol. 13, $\mathrm{n}^{\circ}$ 3, 2013, p. 393-401.

22. Sur la théorie blanchotienne de la parole comme retrait, voir J.-L. Nancy, The Birth to Presence, B. Holmes (trad.), Stanford, Stanford University Press, 1994. La théorie de l'espace personnel comme "cérémonie » est une idée de E. Goffman: Interaction Ritual - Essays on Face-to-Face Behavior, New York, Pantheon, 1982. 
monde, mais ce n'est pas le plus significatif. Ce qui l'est davantage est le rôle que joue la science en venant garantir l'exigence que soit généralement adoptée une nouvelle vision du monde en contraste avec l'ancienne ${ }^{23}$. Cela constituait en soi-même un rôle profondément nouveau pour la science. Dans la période aristotélicienne, la science était comprise comme une forme auxiliaire de la philosophie à l'appui d'une société qui était vouée à la croyance traditionnelle en un créateur suprême. Même la recherche cartésienne de certitude continuait à caractériser la science sous ce jour. Le néoplatonisme garantissait que peu de choses avaient changé dans l'approche de la science à la Renaissance, et cette dernière jusqu'à l'époque de Leibniz procédait de l'affirmation fondamentale de l'existence de Dieu comme d'un premier principe logique ${ }^{24}$.

Le désir de connaître le plan divin, c'est en ce sens que Leibniz lui-même concevait encore ses propres travaux, était pour lui l'essence même du rationalisme philosophique. C'est en opposition avec la séparation plus stricte de la science et de la religion que nous associons actuellement à Francis Bacon. Mais Leibniz lui-même a épousé la méthode de raisonnement fondée sur l'observation. Mieux, les habitants de l'utopie baconienne de Bensalem dans sa Nouvelle Atlantide ${ }^{25}$ étaient convertis au christianisme. Ils professaient le vœu de distinguer les miracles de la réalité. Pourtant ils affirmaient leur désir de connaître les secrets cachés derrière les miracles, non pour les saper, comme le fit David Hume, mais plutôt pour rendre leurs propres résultats aussi convaincants que les illusions divines.

Sans doute, le désir de tirer profit d'un sondage pour ainsi dire divin, qui ne soit pas pur et simple empirisme, a joué un rôle central dans la déconstruction du paradigme aristotélicien ${ }^{26}$. Dans ce dernier, la science avait été le produit de la société; les systèmes de croyance, et non les sciences, fournissaient les perspectives depuis lesquelles les êtres humains pouvaient voir et voyaient le monde; la science servait simplement à placer les objets sous un jour plus clair que celui des croyances. Par contraste, dans le nouveau paradigme scientifique qui plaçait désormais ces systèmes de croyances - l'esprit et les miracles divins - sur une table d'examen, la

23. R. DeWitt, Worldviews: An Introduction to the History and Philosophy of Science, $2^{\mathrm{e}}$ éd., Oxford, Wiley-Blackwell, 2010.

24. L'histoire de la science montre que le concept de Dieu ne relevait pas seulement du contexte culturel de la science à ses débuts, mais était une prémisse des premiers raisonnements scientifiques (ibid.).

25. F. Bacon, The New Atlantis, Seaside, Watchmaker Publishing, 2010.

26. Par exemple, une quête intensément spirituelle anime l'œuvre de Johannes Kepler et pourtant son œuvre de physicien est reconnue comme l'origine clé de la modernisation de la science (voir R. DeWitt, Worldviews...). 
science commençait à rassembler, recueillir les imaginations du peuple avec l'ambition de fournir des visites guidées de l'univers et de révéler un avenir lumineux. Tarde était un héritier de cette nouvelle tradition et il clarifia ses buts de bien des façons: non pour isoler de Dieu la Nature, mais bien pour dépasser l'assujettissement humain à la croyance en une réalité extérieure telle que Dieu ou la Nature (ou la Société durkheimienne). Quoi qu'il en soit, comme nous le verrons, l'interprétation tardienne de ce projet de futurologie à l'état naissant, en termes de déploiement de différences plus hautes ou plus fines (à partir de différences plus basses et moins fines), fera tomber les limites imposées par sa tendance utopique.

Dans Les deux sources de la morale et de la religion, Bergson écrit que les hommes ne réalisent pas suffisamment que leur avenir est dans leurs propres mains, et que l'univers est une «machine à produire des dieux» :

L'humanité gémit, à demi écrasée sous le poids des progrès qu'elle a faits. Elle ne sait pas assez que son avenir dépend d'elle ${ }^{27}$.

Bien que Bergson lui-même fût incertain du rôle futur de la spiritualité - il semblait nourrir quelque espoir en la venue d'un messie ${ }^{28}$-, le point qu'il soulève en commun avec Tarde garde sa pertinence : à savoir que la science (selon l'idée tardienne) ne consiste pas simplement à briser de telles illusions, mais bien à en apprendre les secrets. En tant que penseur du XIXe, Tarde utilise fréquemment le terme de "civilisation». Sous sa plume, la «civilisation » signifie une sorte de vision du monde ou de grande perspective. Détricotant des centaines d'années de néoplatonisme dans une expérience de pensée, il évoque une nouvelle période, un mouvement souterrain qui dépeint le résultat d'une évolution nous conduisant d'un univers saisi par la science à un univers qui peut désormais être aussi scientifiquement produit et maintenu comme un constant "work in progress", sans reposer sur des mythes de pures externalités comme Dieu ou la Nature ou la Société ${ }^{29}$. Comment cela marchera, telle est son expérience de pensée. S'agissant de la courbe du développement de la science, l'univers commence comme un ensemble d'objets à découvrir et expliquer, et s'achève en un monde d'activité humaine et d'expériences à intensifier.

La pensée de Tarde est un produit du XIX ${ }^{\mathrm{e}}$ siècle, mais à de nombreux égards l'activisme et l'anti-représentationnisme propres à son idée de la science commencent seulement à s'imposer. C'est un truisme d'affirmer

27. H. Bergson, The Two Sources of Morality and Religion, Notre Dame (Indiana), University of Notre Dame Press, 1977, p. 317.

28. Ibid.

29. G. Tarde, Underground Man. 
aujourd'hui que la théorie einsteinienne de la relativité a mis au défi la vision mécaniste de l'univers. De façon très récente, les avancées en mécanique quantique ont renforcé l'idée que beaucoup - peut-être la majorité - des principes scientifiques dont nous avons hérité, même ceux relatifs à la théorie de la relativité qu'Einstein tenait pour vrais, sont de naïves illusions. Avec la reconnaissance récente des faits quantiques tels que l'«action à distance», l'utilité de la représentation réaliste des faits physiques et de ses principes, y compris l'utilité du raisonnement causal traditionnel - les images de la réalité -, ont été prises dans les rouets d'un questionnement suspicieux.

Tarde avait prédit que l'activité scientifique ne pourrait être que stimulée et non pas diminuée par cette difficulté de représentation et par les développements scientifiques conséquents qui inviteraient à de nouvelles questions $^{30}$. Par son engagement en faveur du développement d'une méthode scientifique qui n'entame pas le langage de la représentation ${ }^{31}$, la voix de Tarde est optimiste comparée au pessimisme récent devant l'exactitude des faits scientifiques. Deleuze a pointé la formule «chacun sait que» comme la marque même du langage de la représentation ${ }^{32}$. Par exemple, il semble qu'aujourd'hui «chacun sait que» la vision newtonienne du monde était seulement un plateau, et non pas le sommet véritable des exploits humains en matière de physique. Mais notre représentation avantagée par le recul du paradigme mécaniste de la physique envisagé comme un simple tremplin vers l'avenir, trahit parfois trop de suffisance. Certains ont déploré le fait qu'étonnamment, dans notre âge marqué par l'activité scientifique, un nombre significatif d'hommes et de femmes sérieux se sentent en quelque sorte libres de créer leurs propres systèmes de croyances fondés sur de larges représentations de l'univers qui ne correspondent pas à la représentation la plus rationnelle de l'univers que se font la plupart des gens sensés; quelle vérité d'une théorie newtonienne par ailleurs dépassée hante de telles lamentations? On doit pourtant le comprendre. En attendant, deux faits incompatibles, sans rapport apparent, semblent s'être ajoutés, à notre époque, à nos frustrations du moment: les difficultés conceptuelles créées par la physique quantique et le retour d'un certain pittoresque comme une manière de former des idéologies en guise de réponse à cette complexité. Mais ces faits sont-ils sans rapport? Pour

30. G. Tarde, Monadology and Sociology. Voir D. Toews, «Tarde's Sociology of Difference... ».

31. Voir ma discussion de la critique tardienne de la fausseté de l'intellectualisme, qui est symptomatique des problèmes du langage de la représentation: D. Toews, «Tarde’s Sociology of Difference...».

32. G. Deleuze, Difference and Repetition, P. Patton (trad.), New York, Columbia University Press, 1994. 
s'en assurer, on pourrait définir le pittoresque séparément de la science, simplement comme le désir de former des réalités locales, comme une manière de donner du sens à un monde qui semble trop compliqué pour être saisi par des catégories rationnelles ${ }^{33}$. Mais le rôle du pittoresque est-il de simple réaction? Mieux, n'est-ce pas la question de la nature du progrès scientifique qui est devenue cruciale? Des croyances et des systèmes de croyances radicalement différents peuvent-ils s'accommoder d'un consensus sur la méthodologie de la science, à supposer qu'un tel consensus puisse être réalisé, chose en elle-même peu probable?

Anticipant cette question, Tarde met en avant une théorie cosmogénétique $^{34}$ qui, loin d'exclure la notion de pittoresque, l'intègre plutôt. Le pittoresque pour Tarde n'est pas un réalisme local mais désigne plutôt une médiation:

Ce luxe de variations, cette exubérance de fantaisies pittoresques et de capricieuses broderies, que la nature déploie magnifiquement sous son austère appareil de lois, de répétitions, de rythmes séculaires, ne peut avoir qu'une source: l'originalité tumultueuse des éléments mal domptés par ces jougs, la diversité profonde et innée qui, à travers toutes ces uniformités législatives, réapparaît jaillissante et transfigurée à la belle surface des choses ${ }^{35}$.

L'usage tardien du pittoresque assure la médiation entre une réalité phénoménale mondaine faite de représentations conventionnellement et socialement établies, et une réalité nouménale, celle de la différence. Dans la théorie tardienne, le pittoresque désigne simultanément une trame biologique, physique et culturelle de représentation. Similaire à l'univers bergsonien des images, le cosmos est pour Tarde une vision, un panorama sans cesse croissant d'images possibles de la réalité. Aucune image n'est absolue parce que Tarde pose la différence comme la réalité nouménale fondamentale:

En sorte que le sous-sol mystérieux du monde phénoménal serait tout aussi riche en diversités, mais en diversités autres, que l'étage des réalités superficielles ${ }^{36}$.

La différence est nouménale au sens où elle ne peut être représentée directement, et pourtant elle est ce qui rend expressive la signification:

33. Le pittoresque, en ce sens, était une catégorie esthétique majeure du XVIII ${ }^{e}$ siècle. Edmund Burke plaça le terme dans son Enquête sur le beau et le sublime. William Gilpin a lié le pittoresque au paysage.

34. G. Tarde, Social Laws... G. Tarde, The Laws of Imitation.

35. G. Tarde, The Laws of Imitation, p. 72.

36. G. Tarde, Social Laws..., p. 97. 
[...] les originalités sous-phénoménales des choses travaillent non à s'effacer mais à s'épanouir, à éclater en haut ${ }^{37}$.

Aussi les apparences du monde travaillent avec la réalité de la différence, et non pas contre elle, et sont ainsi liées de manière intrinsèque aux sciences qui produisent ces réalités. Leibniz avait décrit un univers composé de monades impersonnelles et sans fenêtres. Dans la pensée néo-monadologique de Tarde, il est plus précis de parler de perspectives d'ordre monadique - ensembles de propriétés symétriques, irréductibles qui font de chaque entité, hypothétique, virtuelle ou réelle, ce qu'elle est. Une perspective d'ordre, dans ce contexte, est comme une logique culturelle, une façon de percevoir le monde ou un système de croyances. En tant que perspectives d'ordre, les monades de Tarde sont reliées les unes aux autres, expriment leur pouvoir relatif les unes sur les autres, leur possession réciproque. J'ai montré que, contrairement à la vision de Bruno Latour s'agissant de l'impersonnalité et de la planéité de ces assemblages, l'analyse de Tarde se fait à partir du point de vue de l'ordre monadique dominant, à savoir, la perception et l'action des êtres humains. Les ordres monadiques sont des logiques culturelles en lutte pour se dominer les unes les autres et ces luttes produisent les expressions que les êtres humains voient comme pittoresques. La puissance d'agir de la composition monadique humaine réside dans sa perception du pittoresque.

Bergson, plus tard, fait écho à ce point quand il fait du corps humain, en un certain sens, une image privilégiée à l'intérieur des images de l'univers ${ }^{38}$. De même, nous pouvons voir que Tarde anticipe la théorie deleuzienne de la représentation molaire: un processus de différenciation produit constamment un conflit entre les ordres monadiques, de nouvelles compositions d'ordres monadiques, et ainsi de nouvelles possibilités de cadres de représentation - ce que Deleuze appellerait «représentations molaires», et ce que Tarde appellerait des possibilités de vues «pittoresques» de l'univers ${ }^{39}$.

Or, pour Tarde, le cosmos se déroule comme un processus de différenciation, comme une production de diversités toujours plus hautes, plus expansives, au grain toujours plus complexe et plus fin ${ }^{40}$. La science est un processus des êtres humains en prise avec cette production de différence

37. G. Tarde, Social Laws..., p. 98.

38. H. Bergson, Matter and Memory, W. S. Palmer, N. M. Paul (trad.), New York, Zone Books, 1988.

39. G. Deleuze, F. Guattari, A Thousand Plateaus: Capitalism and Schizophrenia, B. Massumi (trad.), Minneapolis, University of Minnesota Press, 1987. D. Toews, «The New Tarde: Sociology after the End of the Social», Theory, Culture and Society, vol. 20, $\mathrm{n}^{\circ}$ 5, 2003, p. 81-98.

40. G. Tarde, Social Laws... 
dans l'univers. Tel est le rôle de l'homme dans la cosmogénèse. Notre intention humaine est parfois de ralentir ou de contenir le processus en établissant ce que nous appelons les lois de la nature, mais celles-ci sont seulement des expressions de la cohérence dans la manière dont nous voyons l'univers à un moment historique donné. Les lois de la nature ne sont pas absolues, mais forment plutôt des paradigmes scientifiques qui changent au gré des corrections qui doivent leur être apportées. Tarde établit ainsi que les paradigmes scientifiques évoluent en effet:

Savons-nous les sacrifices de diversités antérieures que la condensation des éléments en atomes similaires, des atomes en molécules et en sphères célestes, des molécules en cellules et ainsi de suite, a exigés au profit de diversités postérieures et, je l'admets, supérieures, ce qui ne veut pas dire accrues ${ }^{41}$ ?

Un paradigme scientifique est ainsi un plateau sur lequel un cadre culturel de représentation de la diversité s'établit pour une période de temps. L'univers progresse à travers des plateaux toujours plus hauts de diversité reconnaissable.

Cependant, parce qu'il est étroitement enchevêtré avec la culture, le progrès scientifique n'est pas une simple progression linéaire. Le développement de l'ordre monadique humain - la possession qui est la nôtre d'ordres monadiques toujours plus nombreux à l'intérieur de notre cadre culturel de représentations, la logique et les fonctions des autres ordres, animaux et géophysiques, que nous avons pris la responsabilité de gérer - complique de façon croissante nos pratiques de représentation, et du pittoresque. C'est William Gilpin qui, à la fin du XVIII e siècle, a théorisé la notion de «pittoresque» comme « un nouvel objet de recherche»; selon lui, l'œil en "quête de beauté» examine le "visage d'un pays", et cet exercice, exigeant que l'on évite le bruit des "grandes usines métallurgiques», est une sorte de chasse ou d'aventure par laquelle on ajoute de la grandeur aux compositions de la nature en proposant des vues en perspective, des cadres de référence singuliers susceptibles de représenter des moments paisibles et apaisants, des moments de simplicité significative ${ }^{42}$ : «Les héros modernes par conséquent ne doivent pas s'attendre à voir leurs exploits immortalisés sur la toile», parce que ce qui devrait être enregistré sur la toile ce sont des réalités alternatives apaisantes qui semblent établir ou venir confirmer une séparation vis-à-vis des uniformités envahissantes de la première

41. G. Tarde, Monadology and Sociology, p. 39.

42. W. Gilpin, Observations on the River Wye [Londres, R. Blamire, 1789], Ann Arbor, University of Michigan Library, 2011, p. 123, en ligne à l'adresse suivante: http://name.umdl.umich. edu/004863361.0001.00o. 
modernité, même si elles positionnent le spectateur comme moderne en glorifiant les ruines d'un passé plus simple ${ }^{43}$. Dans cette séparation du moderne vis-à-vis des espaces sublimes tels que la «pastorale» et la «terre sauvage» (wilderness), ce qui importe est bien sûr toujours de produire activement de telles illusions.

Les « espaces sublimes» ainsi nommés comme la campagne pastorale et la «terre sauvage», apparemment séparés de la modernité, sont néanmoins des notions qui dépendent de l'expansion de notre étonnement et de notre curiosité, lesquels se développent à partir de nos pratiques de la science, étendues et appliquées par l'esprit d'innovation dans la technique artistique. Que les plateaux scientifiques subissent constamment des changements en termes de corrections de nos hypothèses de base, voilà qui garde toute chose en mouvement. Ces changements à leur tour ont des conséquences de plus en plus larges sur l'expérience humaine. Mais la science ne change pas simplement les conceptions humaines de la réalité. Elle change aussi les structures sociales de telle sorte que ces dernières deviennent de plus en plus adaptatives et discriminantes, en termes de reconnaissance de la différence et de la diversité dans l'univers et «dans la nature». Même les réactions politiques d'intolérance et les sentiments opposés à la diversité le reconnaissent implicitement.

Aujourd'hui, le pittoresque a implosé dans les formats des technologies des médias numériques, en permettant au désir de différence et de diversité de croître de façon exponentielle, ce dont témoignent, par exemple, le très grand nombre, la fréquence et la diffusion de données - par exemple les photos digitales - qu'aujourd'hui nous passons notre temps à produire et à manipuler. Tandis que nous atteignons des plateaux de diversités de plus en plus hauts à travers la science et la technologie et des données de plus en plus grosses, la nature d'une "hypothèse de base» dans la science est elle-même de moins en moins capable d'être décrite à la manière du réalisme. Comme l'affirme DeWitt:

L'univers suggéré par les récents développements est un univers qui ne se laisse résumer par aucune métaphore convenable. Il se peut que nous vivions dans un univers qui est... différent de tout ce qui nous est familier ${ }^{44}$.

Comme cela a déjà été noté par nombre de sociologues dès le début $\mathrm{du} \mathrm{XX}^{\mathrm{e}}$ siècle, les processus de la science et de la culture font de moins en moins partie du sens commun. La contribution de Tarde à cette littérature

43. W. Gilpin, Observations on the River Wye, p. 123.

44. R. DeWitt, Worldviews..., p. 348. 
consiste à mettre en lumière le fait que, plutôt qu'elle ne produit des gains en termes de réalisme toujours plus grand, il est plus précis de dire que la science produit toujours plus activement notre socialité.

\section{La futurologie de Tarde}

En plusieurs endroits de ses œuvres théoriques comme de ses œuvres de fiction, Tarde revient sur le thème du futur. Il postule que le futur est susceptible de représenter une forme plus haute de diversité. Les théories sociales de l'homogénéité, de la rationalisation, etc., fondées sur l'idée de l'élimination de la différence, tendent à négliger la dynamique cosmogénétique de l'invention et de l'imitation. En plaçant l'analyse de cette dernière au centre de la théorie sociale, Tarde met en avant (notamment dans les pages finales des Lois de l'imitation dont la citation au début de cet article est extraite) que la destruction de la diversité se révèle comme une étape dans la création d'une diversité plus haute. Étrangement, Tarde démontre que destruction et création impliqueront logiquement la fin de la sociabilité normale et inaugureront une nouvelle période dans laquelle on réévaluera la misanthropie, comme si l'insociabilité était appelée à devenir un principe positif nécessaire à la «personnalité» et à «l'individualité» dans l'âge futur des plus hautes diversités ${ }^{45}$.

Comme nous le verrons, la futurologie tardienne est la clé pour comprendre le jugement prophétique de Tarde à propos d'une misanthropie à venir. Ce que j'appelle la futurologie de Tarde ne peut pas être une futurologie moderniste typique. Tarde a effectivement écrit une œuvre de fiction intitulée Fragment d'histoire future ${ }^{46}$. Elle est construite clairement comme un roman utopique, mais je voudrais montrer que l'orientation de Tarde n'est pas celle de l'utopie moderniste. Dans une telle approche (moderniste typique), le temps et le futur ont été habituellement interprétés en termes bien particuliers ${ }^{47}$. L'orientation temporelle de l'utopie moderniste devrait être envisagée de façon critique dans la mesure où elle implique de dépouiller le passé « de sa validité propre en en faisant le véhicule pour atteindre l'état promis des choses qui est encore à venir ${ }^{48}$. L'approche utopique moderniste implique «de concevoir chaque chose passée comme le tremplin vers le présent et toute chose présente comme

45. G. Tarde, The Laws of Imitation.

46. G. Tarde, Underground Man.

47. Les commentaires généraux que je fais dans cet article sur les utopies s'appliquent également aux dystopies.

48. H. Jonas, The Imperative of Responsibility: In Search of an Ethics for the Technological Age, Chicago, University of Chicago Press, 1985, p. 17. 
le tremplin vers le futur ${ }^{49}$. Néanmoins, des sociologues critiques ont trouvé les causes profondes des tendances utopiques dans la fonction anthropologique des pratiques modernes de communication. Prenant une position similaire à celle d'Habermas, Karl-Otto Apel prétend, par exemple, qu'afin de développer des pensées et des positions valides, il est nécessaire pour les êtres humains d' "anticiper contrefactuellement une forme idéale de communication et par là même d'interaction sociale $»^{50}$. La littérature utopiste moderniste classique est en effet argumentativement et contrefactuellement structurée. Ainsi, il semble que la tendance moderniste utopique puisse être définie par sa projection vers l'extérieur de préoccupations s'enracinant dans des problèmes de communication.

Il est facile d'admettre que la communication est souvent au premier plan des préoccupations humaines, et ainsi compréhensible que la futurologie moderniste soit une préoccupation constante de beaucoup de penseurs. Cela concorde avec des romans utopistes classiques comme celui de Wells, Une utopie moderne ${ }^{51}$, qui néglige de problématiser la position du voyageur et narrateur de l'utopie (celle que Wells dans son récit, en un accès de littéralisme, appelle simplement «le propriétaire de la voix»). Devrionsnous réduire toute futurologie à une anthropologie universelle et positive? N'est-ce pas plutôt l'inverse? Ne devrions-nous pas voir la futurologie plutôt comme une tentative de transcender la condition humaine présente, comme cela semble clair en particulier dans les ouvrages des années 1960 ? Les meilleurs auteurs de science-fiction et œuvres d'anticipation publiés à partir des années 1960 n'étaient-ils pas des visionnaires de la différence par excellence? La science-fiction et le fantastique sont-ils toujours enracinés dans les problèmes de communication du présent, nous renvoyant toujours et encore à une politique de l'identité et de la voix, ou est-il possible d'envisager une futurologie qui impliquerait des formes imaginatives de connaissance entraînant des types d'interaction sociale indifférents aux problèmes de la communication de cette connaissance, et ainsi plus radicalement distinctes du présent - à l'instar des rêveurs lucides d'Ursula Le Guin sur la planète de Nouvelle-Tahiti ${ }^{22}$ ? Le passé et le présent sont inévitablement déformés quand l'histoire est biaisée en faveur des affirmations culturelles présentes d'un narrateur. L'affirmation selon laquelle la communication est le mode dominant de l'interaction sociale

49. H. Jonas, The Imperative of Responsibility..., p. 16.

50. K.-O. Apel, «Is the Ethics of the Ideal Communication Community a Utopia? On the Relationship between Ethics, Utopia, and the Critique of Utopia ", in The Communicative Ethics Controversy, S. Benhabib, F. Dallmayr (dir.), Cambridge, MIT Press, 1990, p. 46.

51. H. G. Wells, A Modern Utopia, Auckland, The Floating Press, 2009.

52. U. K. Le Guin, The Word for World Is Forest, Londres, V. Gollancz, 1977. 
n'est-elle pas la source d'une telle distorsion? La tentative moderniste de saisir le futur dans ses propres représentations, ancrées dans le présent, ne fausse-t-elle pas le futur en même temps que le passé et le présent, et les meilleures œuvres de science-fiction et histoires fantastiques ne sont-elles pas précisément des tentatives d'imaginer des futurologies alternatives? Les préoccupations du temps présent tournent-elles autour de l'imaginaire communicationnel universel et anhistorique, avec toutes les temporalités jugées à l'aune d'un tel critère? Ou bien le futur et le thème de l'alien ne devraient-ils pas plutôt être conçus comme une transformation des priorités humaines?

Si l'impulsion utopique est conditionnée culturellement par les préoccupations de notre présent, pour les mêmes raisons, l'établissement d'un nouveau genre de futurologie informée par la pensée de Tarde, enracinée dans l'idée de diversités progressives plus hautes, ne peut pas y participer en tenant les représentations du passé et du présent pour acquises. Le problème soulevé, pour commencer, est celui du critère utilisable pour évaluer nos relations avec le passé, le présent et le futur. Ce que je veux suggérer, c'est que Tarde en donne un aperçu en montrant comment le problème du temps et du futur est intimement lié à la question de la sociabilité. La sociabilité est un concept lié à la construction discursive de la vie sociale, qui commençait à émerger à la fin du siècle. Comme le montrent les œuvres de Baudelaire, l'indice de vitalité intensive de la sociabilité devient un indicateur du progrès moderne, dès lors que ce dernier a le pouvoir de façonner la sociabilité, par exemple d'éliminer de plus vieilles formes de sociabilité et d'en instituer de nouvelles ${ }^{53}$.

Tarde analyse la sociabilité en mettant l'accent particulièrement sur la conversation. À première vue, la définition tardienne de la conversation comme une espèce d'interaction sociale complète l'image de Simmel de la «bonne forme sociable " ${ }^{54}$. Pour Simmel, la sociabilité élève le bien social, et fait de l'insociabilité un écart vis-à-vis de telles normes. Il élucide ce concept en employant la distinction kantienne entre une "fin en soi» et un "moyen en vue d'une fin", en associant fermement la sociabilité à la première. Ainsi se forge une image de la sociabilité comme pratique désintéressée. En tant que "fin en soi», la sociabilité de Simmel implique des interactions non structurées avec les autres, spontanées, joyeuses, et réjouissantes. La sociabilité «dans sa forme pure n'a aucune fin ultérieure, aucun contenu, et aucun résultat hors d'elle-même [et est] orientée complètement vers les

53. C. Baudelaire, The Painter of Modern Life and Other Essays, J. Mayne (éd. et trad.), Londres, Phaidon Press, 1970.

54. G. Tarde, «The Public and the Crowd». 
personnalités " 55 . De plus, la sociabilité est fondée en prémisses sur l'égalité provisionnelle ou virtuelle de ses participants ${ }^{56}$. Simmel pose ainsi que la «bonne forme» dans la sociabilité de chacun est d'une grande importance ${ }^{57}$. Elle permet la «mutuelle définition de soi [...] à travers laquelle une unité est faite $»^{5}$. Elle implique une situation de jeu dans laquelle les acteurs " "jouent" réellement la société »59. Avec une telle vision de la sociabilité, Simmel a ainsi l'intention d'isoler le social dans le «jeu pur et abstrait de la forme ${ }^{60}$. La sociabilité, conclut-il, est le jeu ou la stylisation qui tire nos associations hors du chaos et leur donne quelque forme. Celle-ci est vue en définitive comme la source de l'unité sociale et la condition sine qua non de possibilité de tout ordre social.

De façon similaire, pour Tarde, la conversation désigne «tout dialogue sans utilité directe et immédiate, où l'on parle surtout pour parler, par plaisir, par jeu, par politesse ${ }^{61}$. Pour Tarde, la conversation s'appuie sur la coprésence, puisqu'elle est une occasion d'observer « un individu dans toute la force de son attention ${ }^{62}$.

Cela, à son tour, mobilise énergiquement les instincts imitatifs qui permettent la diffusion des «sentiments, idées et modes d'action " dans une société moderne ${ }^{63}$. Pourtant, contrairement à Simmel, Tarde ne lie pas la conservation au jargon kantien des fins, dans lequel la sociabilité est strictement vue comme une "fin en soi» ${ }^{64}$. Tarde dresse plutôt une distinction intéressante et utile entre les conversations obligatoires et volontaires ${ }^{65}$. Les conversations obligatoires ont lieu dans le contexte d'une relation de pouvoir entre un inférieur et son supérieur. Leurs origines sont les cérémonies prémodernes du don de cadeaux dans lesquelles les chefs recevaient des présents de leurs vassaux ou autres seigneurs, où il y avait toujours un échange long impliquant des compliments étendus et des remerciements; ces traditions ont passé à travers les générations et sont devenues une forme culturelle distincte d'expression ${ }^{66}$.

55. G. Simmel, «The Sociology of Sociability», American Journal of Sociology, vol. 55, $\mathrm{n}^{\circ} 3$, 1949, p. 255.

56. Ibid., p. 257.

57. Ibid., p. 255.

58. Ibid.

59. Ibid., p. 258.

60. Ibid., p. 255

61. G. Tarde, "Opinion and Conversation", in Gabriel Tarde on Communication..., p. 308.

62. Ibid.

63. Ibid

64. G. Simmel, «The Sociology of Sociability».

65. G. Tarde, «Opinion and Conversation», p. 311.

66. Ibid., p. 307-318. 
Dans l'analyse tardienne, les conversations volontaires (facultatives), contrairement aux conversations obligatoires, se déroulent entre égaux ${ }^{67}$. Par exemple, des amis ou collègues peuvent avoir une conversation manifestant « un souci très vif de la précieuse existence de celui à qui l'on parle, ou un désir intense de le revoir ${ }^{68}$. L'idée d'une conversation volontaire est clairement plus moderne. Pourtant, en même temps qu'il décrit les conversations volontaires comme plus librement sociables que ce qui avait lieu dans les temps passés quand le soi n'était pas cultivé, Tarde attire l'attention sur la relation remarquable entre les intérêts de pouvoir et les pratiques de la conversation volontaire. Il souligne que «c'est donc des monologues prononcés par les supérieurs que s'alimentent les dialogues entre égaux ${ }^{69}$. Et de plus, «il est très rare que les rôles soient d'une égalité parfaite » chez les interlocuteurs dans ces conversations volontaires, ce que l'on voit clairement dans la plupart des cas où «l'un parle beaucoup plus qu'un autre ${ }^{70}$.

L'analyse de Tarde nous aide à éviter un possible contresens: une «bonne forme sociale» n'est jamais «bonne» dans un sens strictement éthique. Elle est bonne plutôt si, au moins nominalement, elle accroît la solidarité sociale. L'analyse simmelienne de la sociabilité soutient que sa fonction est de produire l'unité sociale. Pourtant, l'analyse de Simmel est comparativement anhistorique. Par opposition, celle de Tarde nous permet de percevoir que ce qui prépare le terrain à la dissolution de la «bonne forme sociable» comme véhicule obligatoire de la reconnaissance et du pouvoir social associés aux sociétés prémodernes, c'est la dissimulation décroissante de l'insociabilité - «vraiment pourquoi devrais-je cacher ma peur, mon ennui, mon anxiété ?» - qui vient avec le désenchantement du monde associé à l'essor de la modernité industrielle. Quand Simmel analyse les interactions sociales dans les villes modernes qu'il décrit comme encombrées et submergeant l'individu, il pointe exclusivement une réponse psychologique: la formation du tempérament «blasé». Par opposition, l'analyse tardienne des mêmes environnements urbains souligne le fait que l'hypersociabilité dans la ville s'accompagne d'un rôle positif croissant de l'intimité et de l'insociabilité, et en réalité d'une dynamique de l'interaction sociale dans laquelle des moments insociables impliquant le besoin pour le soi de prendre de la distance vis-à-vis des autres sont requis pour la

67. «Et l'égalité des hommes favorise ses progrès [de la conversation volontaire], autant qu'elle contribue à rétrécir le domaine de l'autre» (ibid., p. 311).

68. Ibid., p. 312.

69. Ibid.

70. Ibid. 
formation des publics, tandis que les impulsions sociables sont satisfaites dans les moments qui impliquent des foules.

Dans la théorie tardienne des interactions sociales au sein des environnements urbains, l'énergie et la vitalité de la foule, en rechargeant les batteries des individus, leur permettent de se retirer à nouveau dans leur «chez soi» privé et autres espaces isolés; après un certain temps, ils reviennent vers la foule pour une nouvelle charge d'énergie ${ }^{71}$. Cette dynamique décrit les rythmes élémentaires de la ville moderne et rend possible le développement du soi sans que soient versées au compte d'une psychologie de l'individu, solipsiste et narcissique, toutes les pressions sociales modernes. La communication est fondamentalement une partie de la dynamique sociale, mais cela n'est pas un fardeau anthropologique et psychologique pour l'individu qui devrait faire un effort pour être sociable en milieu urbain, déjà précisément naturellement excessivement sociable. En comparaison avec la théorie tardienne, celle de Simmel échoue à voir comment le problème d'une excessive sociabilité est pris en compte dans la culture moderne par la création de nouvelles dynamiques sociales et de nouveaux réseaux.

\section{Conclusion}

Tarde nous offre une futurologie qui refuse d'associer l'obligatoire à l'égard du présent et le volontaire à l'égard du futur (ou vice versa comme dans les vues dystopiques), et envisage plutôt les mixtes simultanés et distincts du jeu et de l'obligation, de la sociabilité et de l'insociabilité.

C'est une futurologie à l'écoute de la diversité comprise à la fois comme réalité et production, bien visible dans les analyses relatives à la participation et au détachement des individus et des groupes dans la vie sociale. L'environnement social qui l'illustre le mieux est la sociabilité et l'insociabilité de la ville moderne. Le milieu de Tarde est celui de la sociabilité et de l'insociabilité de la ville moderne; l'insociabilité ici n'est pas une simple aversion pour les autres, mais est plutôt rendue nécessaire par l'hyperactivité et l'excessive sociabilité de l'environnement urbain. La constitution des sociétés modernes n'est ainsi pour Tarde jamais complètement englobée par toutes les sortes d'obligations et contraintes sociales identifiées par Durkheim.

La question de la constitution du collectif qui a nourri le débat avec Durkheim s'infléchit graduellement, dans la pensée tardienne, en une autre

71. G. Tarde, «The Public and the Crowd». 
question, à savoir celle de la relation entre la nouvelle signification de la misanthropie et la science. On pourrait dire que la futurologie de Tarde s'enracine dans une appréciation de la relation du soi avec les autres et avec l'extérieur, la science étant le mode prééminent de cette relation. Sa notion du pittoresque joue ici un rôle crucial car elle témoigne du rôle actif de l'extérieur dans sa pensée, à savoir des environnements irréductibles à la vie sociale humaine mais qui l'informent. Le pittoresque était destiné à élever la représentation des objets et des environnements ordinaires. Comme la science produit une plus haute diversité d'objets, le pittoresque sert de médiation entre une plus haute diversité de ces expériences insondables et des objets modernes complexes. Aujourd'hui les utilisateurs des médias sociaux «capturent» - copient et multiplient - les données. La signification de ces données est, en partie, production: le mode de relation de l'image du soi à toutes les possibilités d'images en général constitue une forme de représentation contingente de diversités, se démultipliant constamment, plutôt qu'une banque passive d'identités. La pensée de Tarde, dès lors, illustre la manière dont le principe de différence dicte la production de réalités.

David Toews

Université de Toronto, Canada

(traduction Anne Devarieux avec l'aide amicale de Thibaut Von Lennep) 
